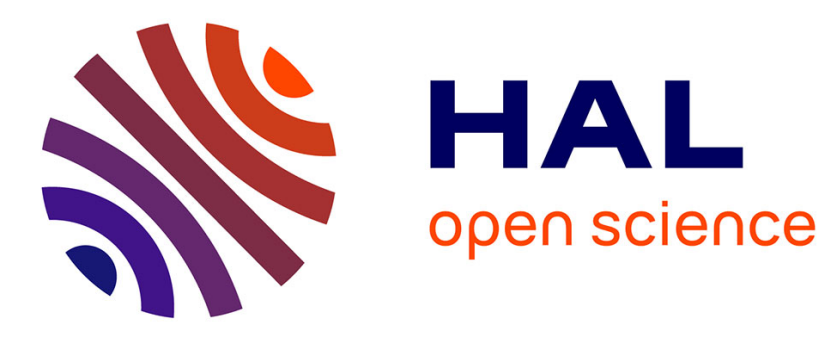

\title{
Reliability and performances of real-time time-dependent auxiliary density functional theory
}

Rika Tandiana, Carine Clavaguera, Karim Hasnaoui, Jesús Naín

Pedroza-Montero, Aurélien de La Lande

\section{- To cite this version:}

Rika Tandiana, Carine Clavaguera, Karim Hasnaoui, Jesús Naín Pedroza-Montero, Aurélien de La Lande. Reliability and performances of real-time time-dependent auxiliary density functional theory. Theoretical Chemistry Accounts: Theory, Computation, and Modeling, 2021, 140 (9), 10.1007/s00214-021-02819-9 . hal-03449111

HAL Id: hal-03449111

https:

\section{//hal-universite-paris-saclay.archives-ouvertes.fr/hal-03449111}

Submitted on 25 Nov 2021

HAL is a multi-disciplinary open access archive for the deposit and dissemination of scientific research documents, whether they are published or not. The documents may come from teaching and research institutions in France or abroad, or from public or private research centers.
L'archive ouverte pluridisciplinaire HAL, est destinée au dépôt et à la diffusion de documents scientifiques de niveau recherche, publiés ou non, émanant des établissements d'enseignement et de recherche français ou étrangers, des laboratoires publics ou privés. 


\title{
Reliability and Performances of Real-Time Time-Dependent Auxiliary Density Functional Theory
}

\author{
Rika Tandiana ${ }^{1}$, Carine Clavaguéra ${ }^{1}$, Karim Hasnaouii $^{2,3}$, Jesús Naín Pedroza-Montero ${ }^{4}$, \\ Aurélien de la Lande ${ }^{1^{*}}$
}

1. Institut de Chimie Physique, CNRS, Université Paris Saclay, Orsay, France

2. High Performance Computing User Support Team, Institut du Développement et des Ressources en Informatique Scientifique (IDRIS), 91403 Orsay, France

3. Maison de la Simulation, CNRS, Commissariat à l'Energie Atomique et aux Énergies Alternatives (CEA), Université Paris-Saclay, 91191 Gif-sur-Yvette, France

4. Programa de Doctorado en Nanociencias y Nanotecnologías,CINVESTAV, Av. Instituto Politécnico Nacional 2508, Mexico

To whom correspondence should be addressed: aurelien.de-la-lande@universite-paris-saclay.fr

\begin{abstract}
We recently adapted the Auxiliary DFT framework as implemented in deMon2k to the simulation of time-dependent problems via the Runge and Gross equations. Our implementation of the so-called Real-Time-Time-Dependent ADFT (RT-TD-ADFT) fully benefits from the algorithms available in deMon2k to carry out variational density fitting, notably the MINRES algorithm recently proposed for self-consistent-field calculations. We test here MINRES for the first time in the context of RT-TD-ADFT. We report extensive benchmarks calculations to assess the reliability of the ADFT framework. These encompass the construction of absorption spectra in the gas phase and in solvent, the calculation of electronic stopping power curves, the irradiation of zeolites by swift ions and the investigation of charge migrations with attosecond time resolution. All our results are very encouraging. We show that even small auxiliary basis sets are sufficient to obtain results almost undisguisable from those obtained with large and flexible auxiliary bases. Overall, we establish the reliability of RT-TD-ADFT to simulate electronics dynamics in large or very large molecular systems.
\end{abstract}




\section{Introduction}

Real-Time Dependent Density Functional theory has emerged in the last two decades as a powerful approach to simulate electron dynamics within molecules, supramolecular assemblies or solids[1, 2]. The method is rooted in the Runge and Gross theorem[3]. At variance to Linear-Response TD-DFT which is a well-established method relying on perturbation theory to simulate absorption spectra of molecules[4], RT-TD-DFT relies on the numerical propagation of the electronic equations-of-motion[5, 6]. It is not limited in principle to perturbations falling into the linear regime. RT-TD-DFT is available in many programs relying either on grid-based[1, 2], on plane waves[7, 8] or on localized basis sets[9-12] with compelling applications to interpret experimental electronic photoelectron spectra, attosecond spectroscopy experiments and pulse radiolysis results, to cite but a few examples[13].

To speed-up RT-TD-DFT simulations, one can resort to well-known methods borrowed from stationary DFT calculations, notably the variational fitting of the Coulomb potential (density fitting, DF) or the Resolution of the Identity (RI). Note that density fitting relies on the variational fitting of the Coulomb potential, a feature that departs from the Resolution of the Identity approach (see [15] for a comparative discussion between the two approaches). A RT-TD-DF-DFT implementation was reported in the NWChem code $[10,14]$ with promising results, while a RT-TD-RI-DFT scheme was reported recently, again showing accuracy of the methodology[11]. We devised on our side an implementation of RT-TD-DFT within the framework of Auxiliary DFT (ADFT) as implemented in deMon2k[16, 17]. In ADFT, auxiliary densities fitted to minimize Coulomb repulsion error are also used in the evaluation of local exchange-correlation energy. The ADFT terminology stems from the fact that the Kohn-Sham potential doesn't depend explicitly on the Kohn-Sham density, but only on auxiliary density[18]. ADFT speedss-up the calculation of electronic integrals and improve scaling with system size $[15,19,20]$. Our implementation of RT-TD-ADFT in deMon2k takes advantage of the most recent algorithmic developments of the code, notably the double-asymptotic integral[21] scheme method to evaluate electronic integrals or the numerical solvers to carry out variational density fitting[22, 23]. These features, combined with an efficient implementation of RT-TD-DFT equations[17], enabled us to tackle electron dynamics simulations in large molecular systems comprised of up to a few thousands of electrons[24].

On the other hand, we have, to our point of view, insufficiently investigated the conditions of reliability of RT-TD-ADFT simulations, in particular the dependence on the quality of the auxiliary basis set. The objective of the present article is to address this point. We will consider the calculation of absorption spectra of molecules in the gas phase and in presence of solvent; the calculation of electronic stopping power in a lithium cluster; the deposition of energy by a $1 \mathrm{MeV}$ protons within a zeolite, and finally charge migrations within an ionized peptide. 


\section{Methods}

\subsection{Real-Time Time-Dependent ADFT}

The simulation of electron dynamics in the framework of Density Functional Theory relies on the Runge and Gross theorem[3] that provides the equations-of-motion for the Kohn-Sham molecular orbitals $\psi_{i}$ (MO):

$i \frac{\partial \psi_{i}(\boldsymbol{r}, t)}{\partial t}=H[\rho(\boldsymbol{r}, t)] \psi_{i}(\boldsymbol{r}, t)$

or, alternatively, if put under the form of a Liouville von-Neumann equation[9, 25]:

$$
i \frac{\partial P(t)}{\partial t}=[H(t), P(t)]
$$

In these equations $H$ is the Kohn-Sham Hamiltonian that functionally depends on the time-dependent electron density $\rho . P$ is the density matrix. In so-called real-time propagation of the electron density we propagate Eq. (1-2) by discretizing time into small time step. We consider in this work the second order Magnus operator[26]:

$$
\begin{aligned}
& P(t)=U\left(t, t_{0}\right) P\left(t_{0}\right) U^{\dagger}\left(t, t_{0}\right) \\
& U\left(t, t_{0}\right)=\exp \left[-i H\left(t+\frac{\Delta t}{2}\right) \cdot \Delta t\right]
\end{aligned}
$$

We express in deMon2k the Kohn-Sham MO as Linear Combination of Gaussian Type Orbitals (LC-GTO). They permit to express the electron density as a sum of products of GTO.

$$
\rho(\boldsymbol{r}, t)=\sum_{\mu, v}^{N_{A O}} P_{\mu v}(t) \mu(\boldsymbol{r}) \nu(\mathbf{r})
$$

We use Greek letters to denote and index GTOs. The elements of the density matrix are evaluated from the complex Kohn-Sham orbitals coefficients. For a closed-shell system we have:

$$
P_{\mu \nu}=2 \sum_{i}^{o c c} c_{\mu i}^{*} c_{v i}
$$

Note that because of the imaginary unit entering eq. 1 , the $\mathrm{MO}$ coefficients are complex numbers in RT-TD-DFT propagations. We now introduce the RT-TD Density-Fitting DFT (RT-TD-DF-DFT) and the RT- 
TD Auxiliary DFT (RT-TD-ADFT) methods. In RT-TD-DF-DFT, a variationally fitted density is introduced to evaluate the classical repulsion term among electrons $[15,20]$. In deMon2k, the fitted density is expressed as linear combination of Hermite Gaussian functions, $\tilde{\rho}(\boldsymbol{r}, t)=\sum_{k} x_{k}(t) k(\boldsymbol{r})$. The $x_{k}$ are the fitting coefficients, which are real quantities. The DF-DFT energy and the Kohn-Sham potential $\operatorname{read}[15]$ :

$$
\begin{aligned}
& E_{\mathrm{DF}-\mathrm{DFT}}=\sum_{\mu, \nu} P_{\mu \nu} H_{\mu \nu}^{c o r e}+\sum_{\mu, \nu} \sum_{\bar{k}} P_{\mu \nu}\langle\mu \nu \| \bar{k}\rangle x_{\bar{k}}-\frac{1}{2} \sum_{\bar{k}, \bar{l}} x_{\bar{k}} x_{\bar{l}}\langle\bar{k} \| \bar{l}\rangle+E_{x c}[\rho] \\
& K_{\mu \nu}=H_{\mu \nu}^{c o r e}+\sum_{\bar{k}}\langle\mu \nu \| \bar{k}\rangle+\left(\frac{\partial E_{x c}[\rho]}{\partial P_{\mu \nu}}\right)
\end{aligned}
$$

In these expressions, $H_{\mu \nu}^{c o r e}$ is a matrix element of the core Hamiltonian, collecting electronic kinetic energy and constant external potential. $E_{x c}[\rho]$ is the XC energy, which has a functional dependence on $\rho$. The || sign denotes the Coulomb operator. We can further use $\tilde{\rho}$ to evaluate the exchange and correlation $(X C)$ contributions leading to the so-called ADFT formalism [18, 19]. This simplification drastically reduces the computational cost of the calculation. Indeed, $E_{x c}$ and the associated potential are evaluated by numerical integration over Lebedev grids centered on atoms[27]. The grid work is greatly simplified in the case of ADFT. DeMon2k also offers the possibility to fit the Fock potential giving access to hybrid or range separated XC functionals at low computational cost[33, 34]. We are currently adapting this methodology to RT-TD-ADFT simulations. In the present work, we will only consider LDA (Local Density Approximation) or GGA (Generalized Gradient Approximation) XC functionals.

Following Dunlap[15, 28], the fitting coefficients ( $\boldsymbol{x}$, bold variables denote vectors) are obtained by minimization of the self-interaction-energy $\varepsilon^{2}=\langle\rho-\tilde{\rho} \| \rho-\tilde{\rho}\rangle$. Substituting the definition of the Kohn-Sham and fitted density into this expression leads to a set the inhomogeneous linear equation system,

$$
\boldsymbol{G} \boldsymbol{x}=\boldsymbol{J}
$$

$\boldsymbol{G}$ and $\boldsymbol{J}$ are the Coulomb matrix and vector, respectively, given by

$$
\boldsymbol{G}=\left(\begin{array}{cccc}
\langle\overline{1} \| \overline{1}\rangle & \langle\overline{1} \| \overline{2}\rangle & \ldots & \langle\overline{1} \| \bar{m}\rangle \\
\langle\overline{2} \| \overline{1}\rangle & \langle\overline{2} \| \overline{2}\rangle & \ldots & \langle\overline{2} \| \bar{m}\rangle \\
\vdots & \vdots & \ddots & \vdots \\
\langle\bar{m} \| \overline{1}\rangle & \langle\bar{m} \| \overline{2}\rangle & \ldots & \langle\bar{m} \| \bar{m}\rangle
\end{array}\right) \text { and } \boldsymbol{J}=\left(\begin{array}{c}
\langle\rho \| \overline{1}\rangle \\
\langle\rho \| \overline{2}\rangle \\
\vdots \\
\langle\rho \| \bar{m}\rangle
\end{array}\right)
$$


The number of rows (or columns) of the $\boldsymbol{G}$ matrix is the number of auxiliary functions. There are usually three to four times that of the number of atomic orbitals. Three methodologies are available in deMon2k to solve this set of inhomogeneous equations. In the analytical method, one inverses the $\boldsymbol{G}$ matrix. This operation becomes cumbersome for very large matrices and two alternative numerical solvers are available. The first one relies on the BFGS (Broyden-Fletcher-Goldfarb-Shanno) quasiNewton approach[29-31]. This approach assumes the $\boldsymbol{G}$ matrix to be positive-definite. In this respect, a truncated eigenvalue decomposition (TED) is performed to ensure positive definiteness[23]. Also, for large matrices, involving ten thousands of auxiliary functions, this initialization step may become impossible due to the cubic scaling coming from the matrix diagonalization, even with modern high performance computers (HPC). Moreover, the use of auxiliary functions with small exponents and high angular momentum, compromises even more the positive definiteness of the matrix which might not be guaranteed leading to numerical instabilities, notably in RT-TD-DFT propagations. To overcome these difficulties a MINRES[22, 32] scheme relying on a preconditioned Krylov subspace method has been recently proposed. The MINRES iterative algorithm permits to deal with systems encompassing more than one hundred thousand auxiliary functions with reasonable timings. To this end, the MINRES algorithm avoids the explicit diagonalization of the $\boldsymbol{G}$ matrix and solves Eq. 9 iteratively using matrixvector products. Also, because the matrix inversion is not needed, it reduces the RAM (Random Access Memory) usage and hard disk storage used for the calculations. It is therefore promising for RT-TDADFT simulations of large systems with thousands of atoms. The introduction of MINRES in deMon2k is recent and we took the opportunity of this article to assess its use in RT-TD-ADFT.

\subsection{Performance and stability of RT-TD-ADFT simulations}

Unless otherwise stated, we used the deMon2k developer version 6.1.6[16] to produce all the results presented in this article. The source code is available on request to the authors. We used the $\mathrm{R}$ project for statistical computing[35] to analyze and produce the data (Figures and Tables) presented in this article.

deMon2k is equipped with an algorithm to automatically generate an auxiliary basis set from the atomic orbital basis set. Auxiliary functions are grouped in sets containing $s$, spd or spdfg Hermite Gaussian functions (Hermite Type Orbitals HTO)[36]. In each set the functions share the same exponents. The notation GEN-An $(n=1,2,3 \ldots)$ means an auxiliary basis set containing only $s$ and $s p d$ while the GEN-An* notation the further addition of $f$ and $g$ functions. The number of exponents $N$ (auxiliary function sets) is determined by the user-defined parameter $n$ :

$$
N=\operatorname{Int}\left(\frac{\ln \left(\beta_{\max } / \beta_{\min }\right)}{\ln (6-n)}+0.5\right)
$$


where $\beta_{\max }$ and $\beta_{\min }$ are respectively the largest and smallest exponents of the primitive in the atomic orbital basis set. The larger the $n$ parameter, the larger the number of exponents. In the following we will consider the GEN-A2, GEN-A2*, GEN-A3 and GEN-A3* auxiliary basis sets. In some instance we shall consider an intermediate GEN-A2 ${ }^{\delta *}$ auxiliary basis constructed from GEN-A2* but from which we removed the g functions. GEN-A2 ${ }^{\delta *}$ only incorporates $s, s p d$ and spdf sets.

To test the performances of RT-TD-ADFT with MINES, we consider as in Ref [22] a series of fullerenes and of water clusters. Simulations have been carried out on the Jean Zay supercomputer hosted at the IDRIS center using 20 nodes. All simulations have been carried out with the PBE[37] XC energy functional with a numerical integration grid of ultrafine accuracy $\left(10^{-7} \mathrm{Ha}\right)$. We have used the DZVPGGA AO basis set and the GEN-A2* auxiliary basis. As seen on Figure 1, the largest systems involve more than 70, 000 HTO. Electronic repulsion integrals have been calculated with the so-called direct scheme (ie. the integrals are not stored in RAM, but are recalculated whenever they are needed) and a multipole expansion for long range integrals[21]. The three main computational demanding tasks are plotted on the graph. The calculation of the Kohn-Sham potential is remarkably efficient even with the largest systems, as well as the basis transformations. The most time-consuming part remains the calculation of the exponential of the Kohn-Sham matrix involved in the propagation of the density. This task is achieved here using the Scalapack library[38]. Further improvement of the code could be obtained by using for instance GPUs, but the results shown in Figure 1 already illustrate the capability of RT-TD-ADFT simulations on very large molecular systems. 

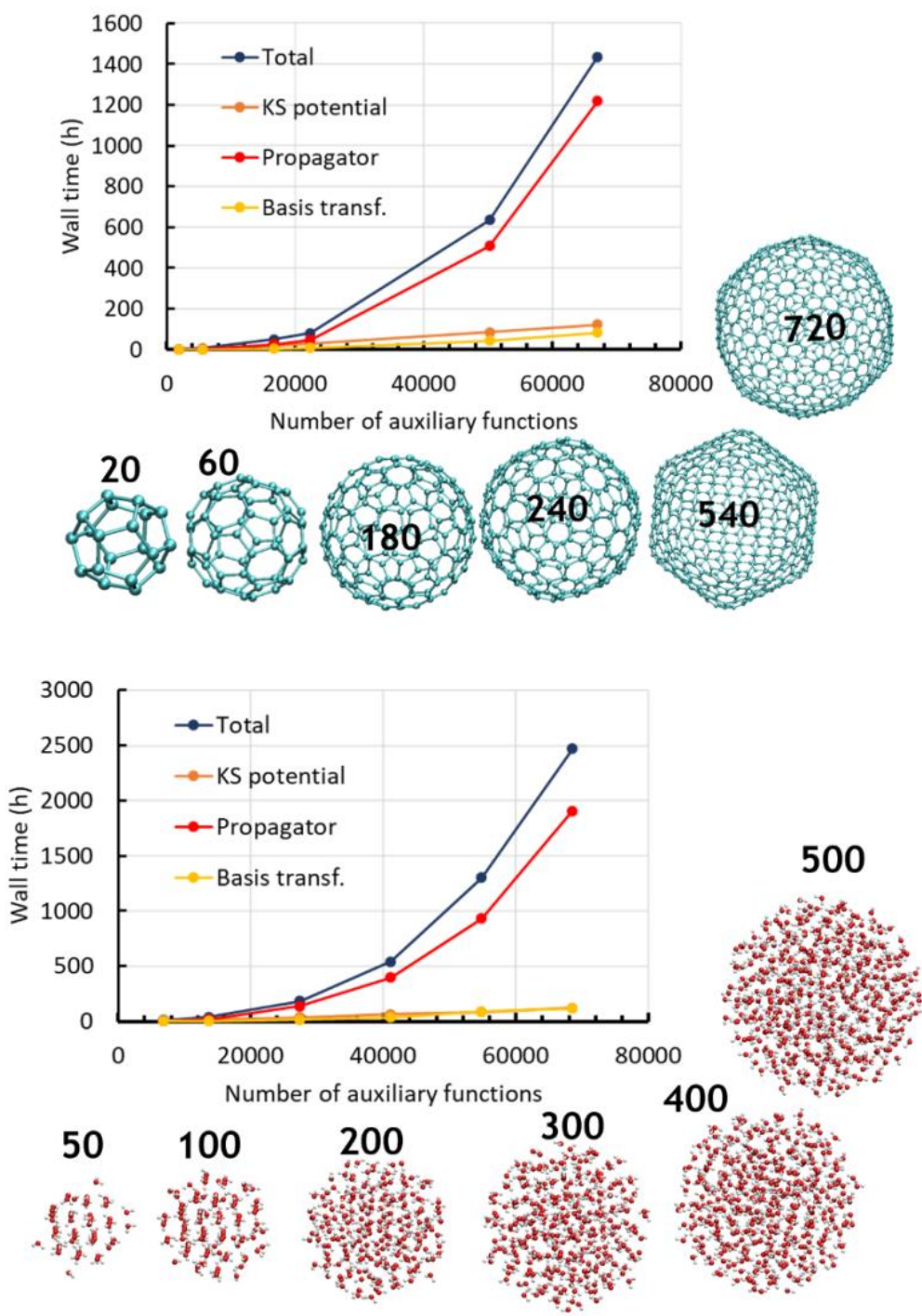

Figure 1: Computational performances of RT-TD-ADFT simulations with MINRES. Wall clock time to conduct 1 fs of RT-TDADFT simulation with a 1 as time step.

To assess the stability of RT-TD-ADFT simulations depending on methodology employed to conduct variational density fitting, we consider a small peptide embedded within a water droplet. We consider two molecular systems. In the first one, the peptide is surrounded by its first hydration layer water, while in the second one a few solvation layers molecules are included. The first and second systems respectively encompass 105 and 465 atoms, for 404 and 1604 electrons. We first converged the SelfConsistent-Field energy minimization with convergence criteria of $10^{-10}$ and $10^{-7} \mathrm{Ha}$ on the MinMax[39] SCF energy and on the Density Fitting error respectively. We used a combination DZVP-GGA[40] and GEN-A2*[41] atomic orbital and auxiliary basis sets. The small and large systems respectively encompass on one hand 1029 and 4029 atomic orbitals and, on the on the hand, 5205 and 22345 auxiliary functions. The large system is large enough but remains amenable to simulations with all three density fitting methodologies. After the SCF, an electric field of strength 0.001 a. u. was applied 
in the $x, y$ and $z$ direction in the first step and we propagated the electronic dynamics for 200 or 100 as. We used an iterative second order Magnus propagation with same convergence criteria as in the SCF, using a timestep of 1 as. After application of the pulse, the electronic system was immersed in a constant potential and its energy should be constant. This is the property we probe here. Table 1 reports the maximum deviation to the mean energy, in absolute value, during the simulation, as well as the time spent to run the simulations. In all cases the simulations were stable with strict conservation of the total number of electrons.

The analytical density fitting produces the most stable propagations with an energy deviation below $5.610^{-9}$ and $3.110^{-8} \mathrm{Ha}$ for the small and large systems respectively. This is in practical applications a good energy conservation that normally ensures reliable propagations. Note that even further better energy stability could be obtained by tightening other simulations parameters such as the accuracy of the $\mathrm{XC}$ integration grid. But for the present discussion the results provided by the analytical density fitting will serve as reference. The BFGS-QN numerical solver based on the SVD decomposition of the $\boldsymbol{G}$ matrix provides very similar results to the analytical solver. The computational advantage of the BFGS-QN appears for the larger system with respect to the analytical algorithm. MINRES with the standard SCF convergence criteria $\left(10^{-8}\right)$ produces slightly less stable simulations, with an energy conservation around $10^{-6} \mathrm{Ha}$. However, when tightening the convergence criteria by a factor of one hundred or so, the energy is conserved up to $10^{-7} \mathrm{Ha}$. The computational cost significantly increases as one tighten MINRES convergence as more cycles are needed to reach convergence. Tolerance of $10^{-10}$ seems to be a good compromise. In this case, RT-TD-ADFT simulations with MINRES are slightly costlier and slightly less stable than those performed with the BFGS-QN numerical solver. However, as outlined above, MINRES permits to address much larger systems than those accessible by the other methods. Therefore, MINRES is extremely valuable to conduct RT-TD-ADFT simulations of large molecular systems.

\begin{tabular}{|c|c|c|c|c|c|c|c|c|}
\hline & \multirow[t]{2}{*}{ Analytical } & \multicolumn{2}{|c|}{ SVD } & \multicolumn{5}{|c|}{ MINRES-TED } \\
\hline & & $10^{-6}$ & $10^{-7}$ & $10^{-8}$ & $10^{-9}$ & $10^{-10}$ & $10^{-11}$ & $10^{-12}$ \\
\hline \multicolumn{9}{|l|}{$5905 \mathrm{HTO}$} \\
\hline Time (s) & 3060 & 3221 & -a & 3191 & 3345 & 3593 & 4355 & -a \\
\hline E. Stability ( $\mathrm{Ha})$ & $5.610^{-9}$ & $5.710^{-9}$ & -a & $9.110^{-7}$ & $1.910^{-7}$ & $3.810^{-7}$ & $8.210^{-8}$ & $-\mathrm{a}$ \\
\hline \multicolumn{9}{|l|}{$22345 \mathrm{HTO}$} \\
\hline Time (s) & 27168 & 14579 & 14492 & 14626 & 15046 & 20083 & $-\mathrm{a}$ & 26807 \\
\hline E. Stability ( $\mathrm{Ha})$ & $3.110^{-8}$ & $2.910^{-8}$ & $2.910^{-8}$ & $3.910^{-6}$ & $4.010^{-7}$ & $2.510^{-7}$ & -a & $1.310^{-7}$ \\
\hline
\end{tabular}

Table 1: Energy stability afforded by the analytical and numerical methods available in deMon2k to carry out density fitting of the Coulomb potential. a. not calculated. 


\section{Results}

In this section we consider various applications of RT-TD-ADFT and we systematically investigate the reliability of density fitting as a function of the auxiliary basis sets.

\subsection{Absorption spectra of coumarine in the gas phase}

We calculate here the absorption spectrum of coumarin in the gas phase. The geometry was preliminary optimized at the DFT level. The principle of the simulation is the following. First we optimized the electron density with convergence criteria of $10^{-10}$ and $10^{-7} \mathrm{Ha}$ on the MinMax and density fitting error respectively. We used an adaptive grid of ultrafine accuracy to integrate the XC contributions, ensuring an accuracy of $10^{-7} \mathrm{Ha}$ on the diagonal elements of the XC potential matrix. We used the PBE functional[37]. Real-time simulations were launched from the SCF solution with application of an electric field kick in either the $x, y$ or $z$ directions. This infinitely narrow electric kick populates all the electronic spectrum and the resulting variations of the molecular dipole moment encodes the energy position of the excited states. The field strength was set to 0.005 a.u. The simulations were run for $10 \mathrm{fs}$ with the predictor-corrector second-order Magnus propagator and a time step of 1 as. To simulate the electron spectrum, we Fourier transformed the dipole moment that was calculated every 1 as. A damping exponential function with a characteristic decay of $4.84 \mathrm{fs}$ was applied on the dipole moment in the post-treatment.

Four auxiliary basis sets have been considered, always in combination with the TZVP atomic orbital basis set. These are GEN-A2, GEN-A2*, GEN-A3 and finally GEN-A2 ${ }^{\delta *}$. They respectively include 508, 1166, 635 and 836 auxiliary functions. The upper panel of Figure 2 addresses the convergence of RTTD-ADFT absorption spectra as a function of the quality of the auxiliary basis set. We shall take GENA2* as reference at it is the richest and most flexible auxiliary basis set. Globally the four curves are extremely similar with extrema located at almost the same wavelengths, indicating a weak dependence on the choice of the auxiliary basis set. The red curve (GEN-A3) is the one that present more pronounced deviations with respect to the green one (GEN-A2*). The deviations remain modest though. Remind that GEN-A3 permits less angular flexibility as it does not include $f$ or $g$ auxiliary functions. On the other hand, the intermediate GEN-A2 ${ }^{\delta *}$, that is similar to the GEN-A2* but deprived from its $g$ auxiliary functions, is also quite satisfactory.

The lower panel compares spectra obtained by the RT-TD-DF-DFT (dashed-dotted lines) and RT-TDADFT (plain lines) approaches for each of the auxiliary basis sets. The agreement between both approaches is remarkable in all cases. Only the spectra obtained with GEN-A3 are somehow a little bit less convincing. These results show that it is safe to use the ADFT formalism to evaluate absorption spectra of organic molecules and that even the GEN-A2 auxiliary basis set has already provided spectra 
extremely close to the computationally more expensive GEN-A2*. We do not have the possibility with deMon2k to perform RT-TD-DFT with four-centers-electron-repulsion-integrals, i.e. without density fitting, but DF-DFT with GEN-A2* leads in general to results very close to pure DFT calculations[18]. We may therefore conclude that RT-TD-ADFT is an excellent approximation to four-centers RT-TD-DFT calculations. In terms of computational efficiency, the reduction of the time afforded by the ADFT formalism over the DF-DFT is of the order of $7,5,5$ and 4 with the GEN-A2, GEN-A2 ${ }^{\delta *}$, GEN-A3 and finally GEN-A2* auxiliary basis sets for the calculation of XC contributions. Overall, the cost of RT-TDADFT propagation, including other tasks, is roughly divided by a factor of 2 .
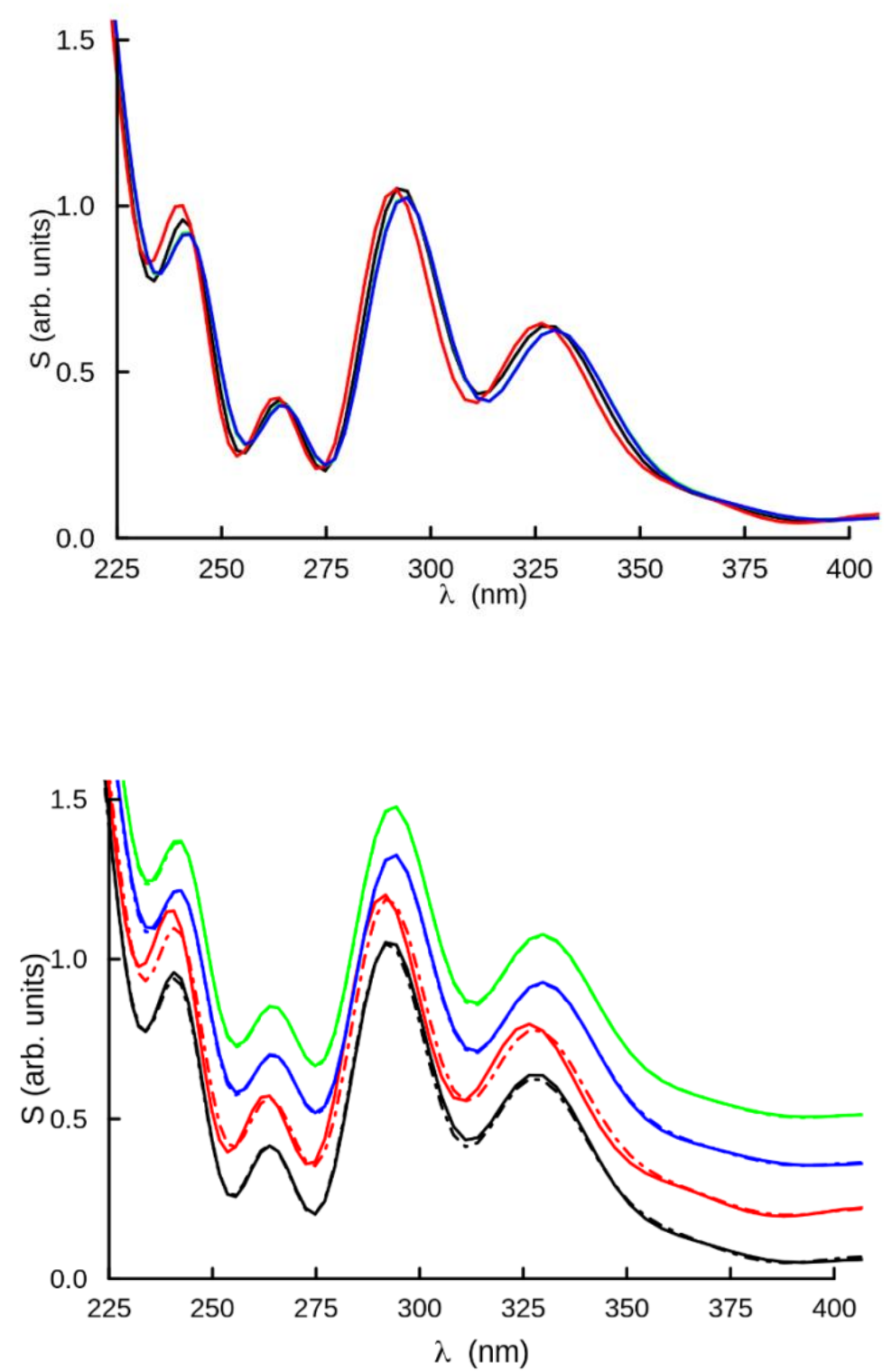

Figure 2: top, absorption spectra obtained from RT-TD-ADFT simulations with GEN-A2 (black), GEN-A2* (green), GEN-A3 (red) and GEN-A2 ${ }^{\delta *}$ (blue). Bottom, spectra obtained with RT-TD-DF-DFT (dashed-dotted lines) and RT-TD-ADFT (plain lines) approaches with different auxiliary basis sets (same color code as top graph). Each pair of curves have been vertically shifted to improve clarity. 


\subsection{Absorption spectra of uracil in water}

We consider here the absorption spectra of the deprotonated uridine monophosphate (USP) in water. We follow a hybrid $\mathrm{QM} / \mathrm{MM}$ approach to account for the water environment. The geometry was extracted from a previous study in which we reported QM/MM MD simulation of this system[42]. The QM system encompasses the deprotonated nucleobase that holds a total charge of -2 . The QM region is placed at the center of a $33 \AA \AA$ radius droplet of water molecules. We have considered the TZVP basis set and four independent auxiliary function sets, namely GEN-A2, GEN-A2*, GEN-A3 and GEN-A3*. Like for the previous section we consider both the RT-TD-DF-DFT and RT-TD-ADFT methods. Simulations have been carried out for $10 \mathrm{fs}$ with the PBE[37] XC energy functional. The electronic spectra are displayed in Figure 3. The dependence of the calculated spectra (Left graph) on the auxiliary basis is very weak. The graph on the right-hand-side of the Figure 2 further shows that no noticeable differences can be detected when using the fitted density in the calculation of XC contributions. Overall the conclusions are the same as in the previous section, namely that the use of the ADFT framework is safe for the calculation of absorption spectra by RT-TD-DFT simulations, together with a very weak auxiliary basis set dependence.
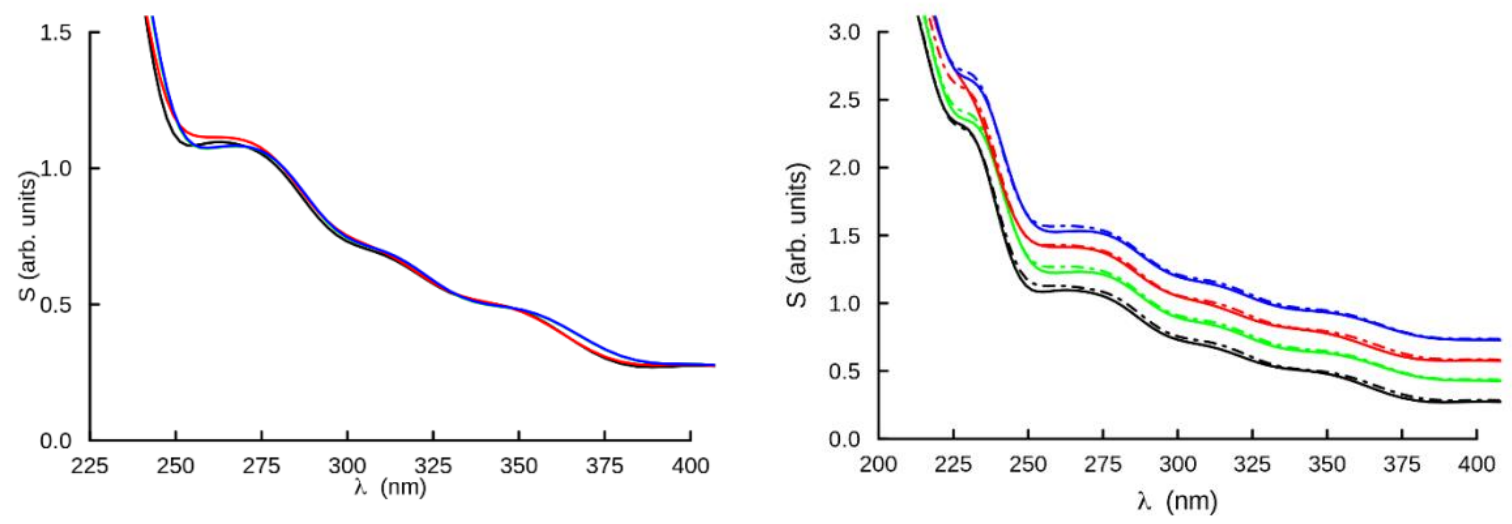

Figure 3: QM/MM absorption spectra of deprotonated uridine monophosphate in water. Left, RT-TD-ADFT spectra with four auxiliary basis sets (GEN-A2 in black, GEN-A2* in green, GEN-A3 in red and Gen-A3* in blue). Right, comparison between RTTD-DF-ADFT (dashed-dotted lines) and RT-TD-ADFT (plain lines) approaches with the four sets of auxiliary functions. For clarity each pair of colored curves has been vertically shifted.

\subsection{Stopping power of proton in aluminum}

We calculate the electronic stopping power curve of proton on a lithium cluster to test the performance of ADFT. The geometry of the cluster, consisting of 62 lithium atoms, has been taken from Maliyov et al., and used without further optimization[43]. Following the conclusion of the authors, we have used two sets of AO basis sets, namely cc-pVTZ for the 18 atoms close to the path of the proton, and cc-pVDZ for the rest of the atoms. Similarly, we have performed the calculation with 
GEN-A2, GEN-A2*, GEN-A3, and GEN-A3* auxiliary functions for both RT-TD-DF-DFT and RT-TD-ADFT frameworks. The criteria for the grid was set to $10^{-8} \mathrm{Ha}$ to ensure accuracy and stability during the following dynamics step. The SCF and CDF criteria have been set to $10^{-9} \mathrm{Ha}$ and $5.10^{-7} \mathrm{Ha}$, respectively. Then, the real time simulation was performed for 500 to $6400 \mathrm{fs}$, depending on the kinetic energy of the projectile, with the predictor corrector second-order Magnus propagator and a time-step of 1 as. The stopping power of the cluster has been calculated as the average of deposited energy with respect to the distance travelled by the projectile within the system $(\mathrm{dE} / \mathrm{dx})$. For our current objective, the electronic stopping power has been calculated from one trajectory as a function of the kinetic energy of proton $(0.005,0.025,0.05,0.1,0.5$, and $1.0 \mathrm{MeV})$ for each of the auxiliary function.

The electronic stopping power curves of lithium cluster calculated with RT-TD-ADFT have been obtained with the four auxiliary basis sets. They include 2 746, 6 620, 3 428, and 8636 auxiliary functions respectively. The curves obtained for each of the auxiliary functions are shown to be overlapping with one another, as seen in Figure 4 (top left). As such in Figure 4 (top right), we show the energy difference between the stopping power curves of each set with the GEN-A3* function, which supposedly is the largest auxiliary sets in the series. The difference is in the scale of $10^{-4} \mathrm{a} . u$., which clearly illustrates the insensitivity of the electronic stopping power to the auxiliary functions used. This has also been shown in terms of the energy deposition $(\mathrm{dE} / \mathrm{dt})$ at maximum deposition at $0.025 \mathrm{MeV}$ in Figure 4 (left). Similarly, GEN-A2 auxiliary basis set performs remarkably well in this system.

Similarly, the electronic stopping power curves calculated with RT-TD-DF-DFT framework for each of the auxiliary functions are overlapping, as seen in Figure 3 (bottom left), with the difference with GENA3* function is also in the range of $10^{-4} \mathrm{Ha}$. (Figure 3 bottom right). As we compare the electronic stopping power curve obtained with both frameworks, they are remarkably similar with differences in the scale of $10^{-4} \mathrm{Ha} /$ bohr as well. Moreover, it is worth mentioning that the RT-TD-DF-DFT framework takes around 3 to 4 times as long for the calculation. Therefore, the performance of RT-TD-ADFT has significantly cut the computational cost, giving access to calculation for larger systems. 

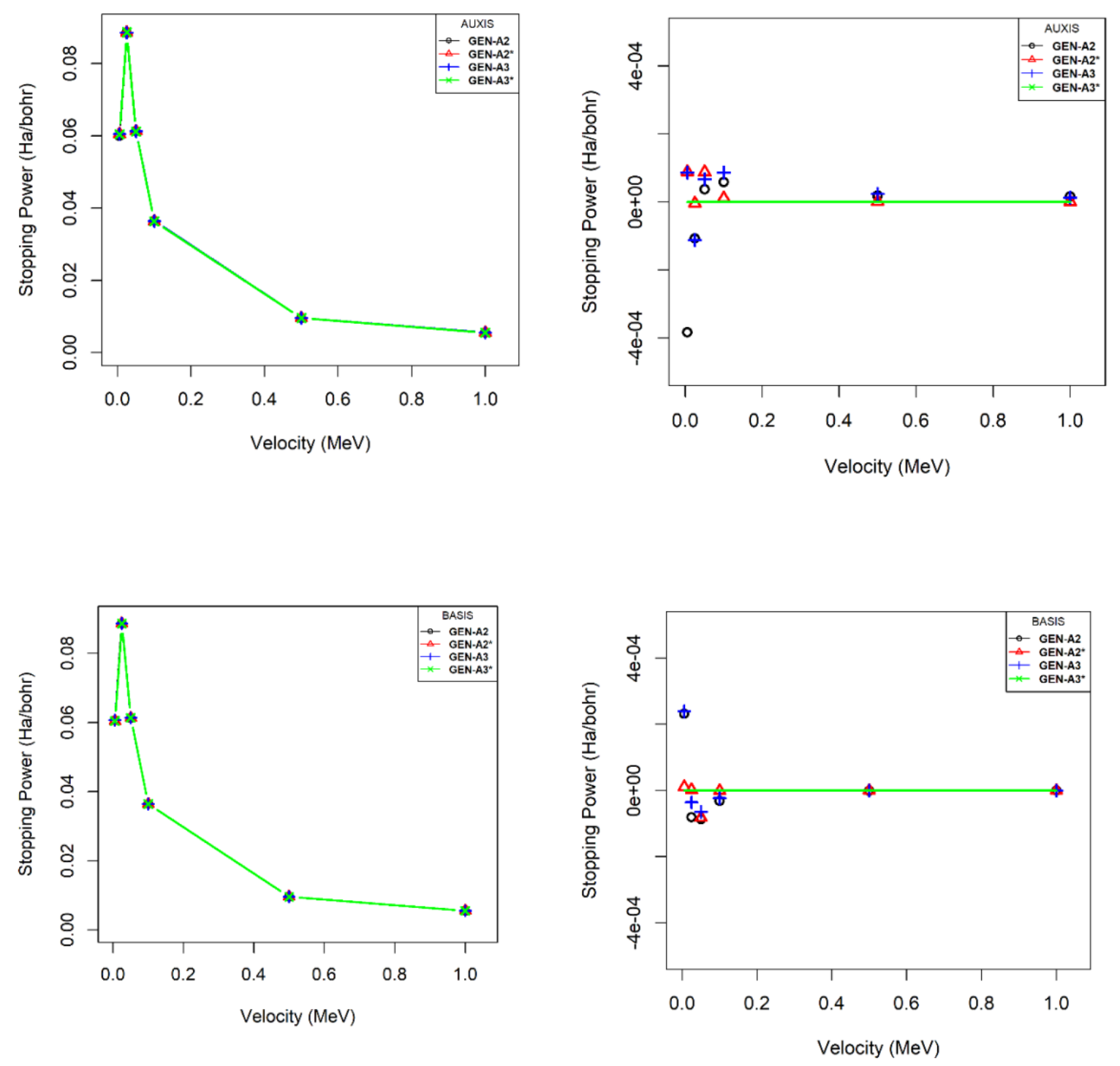

Figure 4: (top-left) The stopping power of proton in lithium as a function of the kinetic energy of the projectile calculated with RT-TD-ADFT (top-right) The difference between the stopping power obtained with the GEN-A2, GEN-A2*, and GEN-A3 with GEN-A3* as the reference. Bottom, same as Top for RT-TD-DF-DFT simulations. 

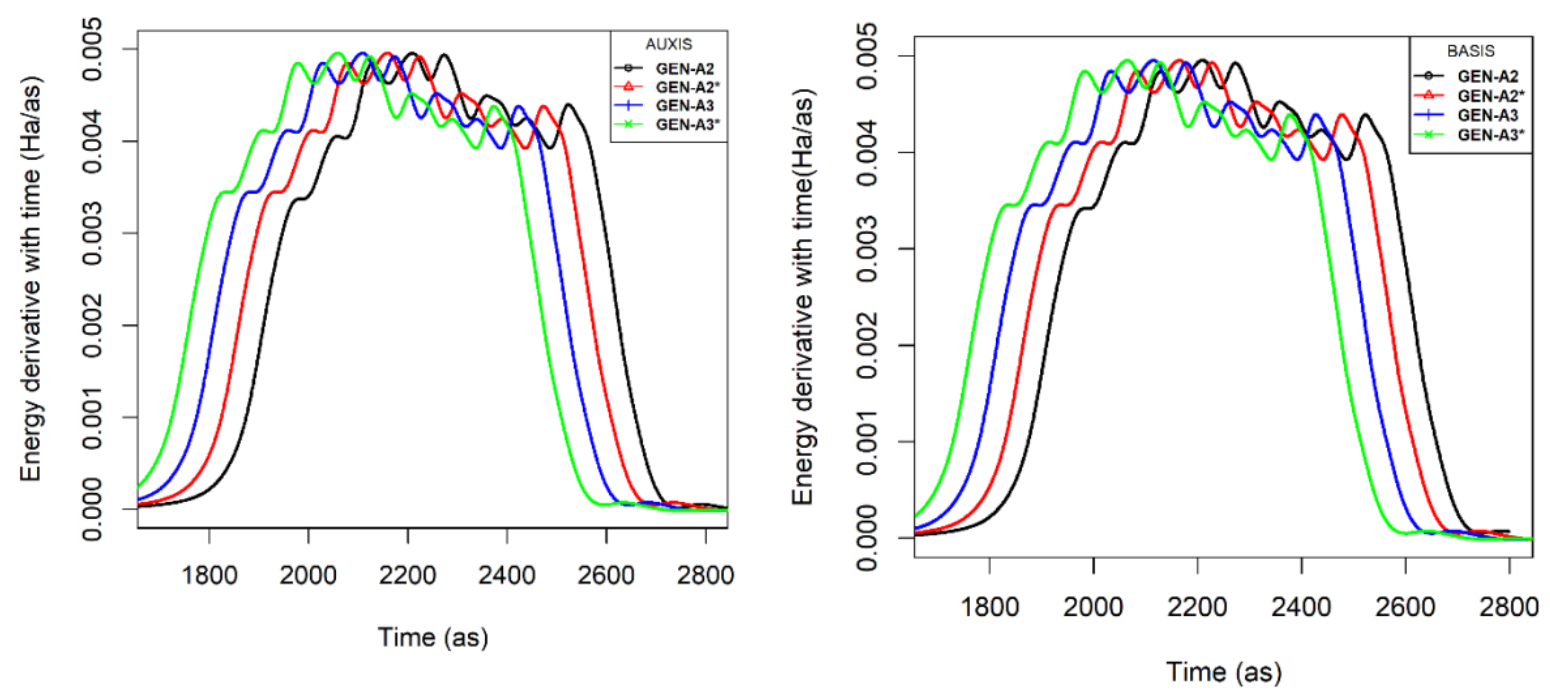

Figure 5: The energy deposition calculated with the series of auxiliary functions at RT-TD-ADFT (left) and RT-TD-DF-DFT (right) as the projectile of $0.025 \mathrm{MeV}$ travel through the lithium cluster. For clarity, the curves have been shifted with respect to the $x$-axis.

\subsection{Charge migrations within a zeolite after irradiation by $1 \mathrm{MeV}$ proton}

RT-TD-DFT simulation is a method of choice to investigate ultrafast electron dynamics following a perturbation by a laser field or the collision with high speed charged particles. The performances of RT-TD-ADFT permit for instance to investigate the deposition of energy within large molecular systems[24]. We consider in this example irradiation of a zeolite by a $1 \mathrm{MeV}$ proton. The system is composed of 483 atoms (126 Si, $287 \mathrm{O}, 70 \mathrm{H}$ ) for a total of 4130 electrons. After convergence of the SCF procedure, we have placed a proton $50 \AA$ away from the center of mass of the zeolite and have launched RT-TD-ADFT simulations. The projectile has a fixed propagation line (along the $x$-axis), and constant speed. We illustrate in Figure 6 the collision conditions. At every time step the position of the projectile is updated. We have chosen the PBE XC energy functional with the DZVP-GGA AO basis set. The XC contribution was evaluated with a grid of high accuracy (ensuring an accuracy of $5.10^{8} \mathrm{Ha}$ on the diagonal elements of the XC potential matrix). We have propagated the RT-TD-ADFT simulations for $1 \mathrm{fs}$ with the predictor-corrector second order Magnus scheme and a Taylor formula to calculate the propagation matrix. We consider four auxiliary basis sets: GEN-A2 on all atoms (BS1), GEN-A2 on $\mathrm{O}$ and $\mathrm{H}$ atoms and GEN-A2* on Si (BS2), GEN-A3 on all atoms (BS3), and finally, GEN-A2 ${ }^{\delta *}$ (BS4) on all atoms. Simulations with BS1, BS2, BS3 and BS4 respectively involve 15 708, 27 426, 20321 and 31339 auxiliary functions. 

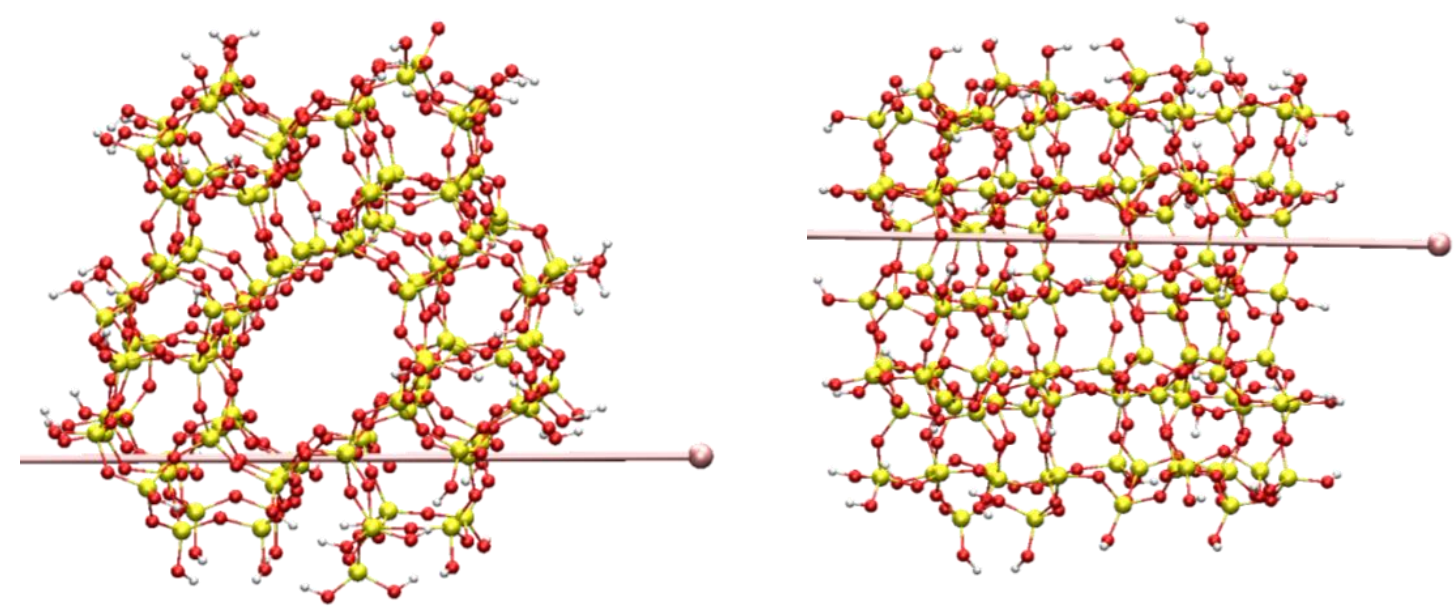

Figure 6: irradiation of a zeolite by a $1 \mathrm{MeV}$ proton. Color code, Si, O and $\mathrm{H}$ in yellow, red and white respectively. The two pictures correspond to a top-view and a side view of the zeolite.

Figure 7 depicts the profile of energy deposition upon collision. The latter takes place 250 as after the beginning of the simulation. The upper panel deals with the RT-TD-ADFT simulation with the largest auxiliary basis set, that we consider here as our reference calculation. Approximately $25 \mathrm{eV}$ are deposited in the electron cloud at the end of the collisional process. This very large number is typical of irradiation by charged particles. The graph depicted on the lower panel shows the difference of deposited energy obtained with the other auxiliary basis sets with respect to BS4. We see that the differences are very small, i.e. two orders of magnitude smaller than the deposited energy. It is worth mentioning at this stage that the total simulation time amounted to 1370, 6912, 3755 and 6435 hours respectively for the BS1, BS2, BS3 and BS4 auxiliary sets. There is thus a factor 4.6 in terms of computational cost between the BS1 and BS4 auxiliary sets for almost undisguisable results as detailed just above.

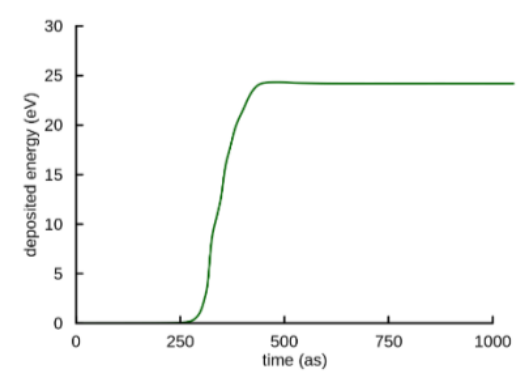




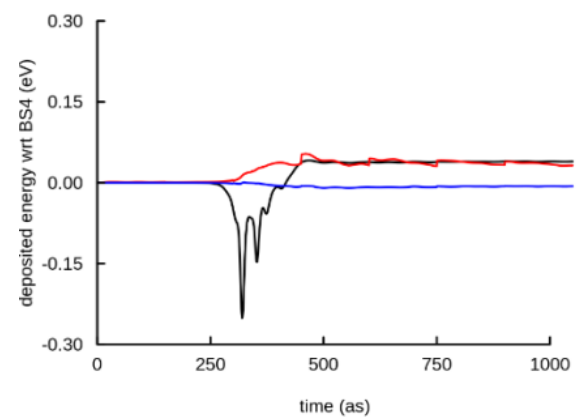

Figure 7: Top, deposition energy profile upon collision of the zeolite with the projectile obtained with the largest auxiliary basis set function (BS4). Bottom: difference of deposited energy with respect to BS4 for the BS1 (black), BS2 (red), BS3 (blue) auxiliary basis sets.

\subsection{Electron flows within an ionized peptide}

In this last application we investigate electron flow within an ionized pentapeptide (Alanine, Alanine, Serine, Alanine, Alanine, see Figure 8). The two extremities of the pentapeptide are modified to accommodate a vinyl chemical group. This molecule was optimized by Berstis et al. as a simple chemical model to study electron tunneling through peptides[44]. During the SCF we impose the sum of the charges on the atoms within the dotted rectangular to be 1 . This is achieved with the constrained DFT module implemented in deMon2k[45]. We then release the constrained and we follow the charge flow by RT-TD-ADFT simulations[46]. We contrast six auxiliary basis sets: GEN-A2 on all atoms (BS1), GEN-A2* on all atoms (BS2), GEN-A2 on $\mathrm{H}$ and GEN-A2* on $\mathrm{C}, \mathrm{N}$ and $\mathrm{O}\left(\mathrm{BS} 3\right.$ ), GEN-A2 ${ }^{\delta *}$ on all atoms (BS4), GEN-A3 on all atoms (BS5) and, finally, GEN-A3* on all atoms (BS6). The systems respectively encompass 1038, 3171, 2631, 2361, 1365 and 4416 auxiliary functions. The DZVP-GGA atomic orbital basis set is used for all atoms. All simulations are carried out with the PBE XC energy functional. We have conducted the RT-TD-ADFT simulations for $10 \mathrm{fs}$ with a time step of 1 as via the predictorcorrector Magnus propagator. Population analyses using Hirshfeld partitioning scheme have been carried out every 50 as based on the fitted density. Figure 9 depicts the charge evolutions following the system decomposition in fragment shown in Figure 8. The fragments roughly correspond to the aminoacid residues, except for the terminal groups that have been modified. We only depict the charges for the first three fragments that exhibit the largest variations. 


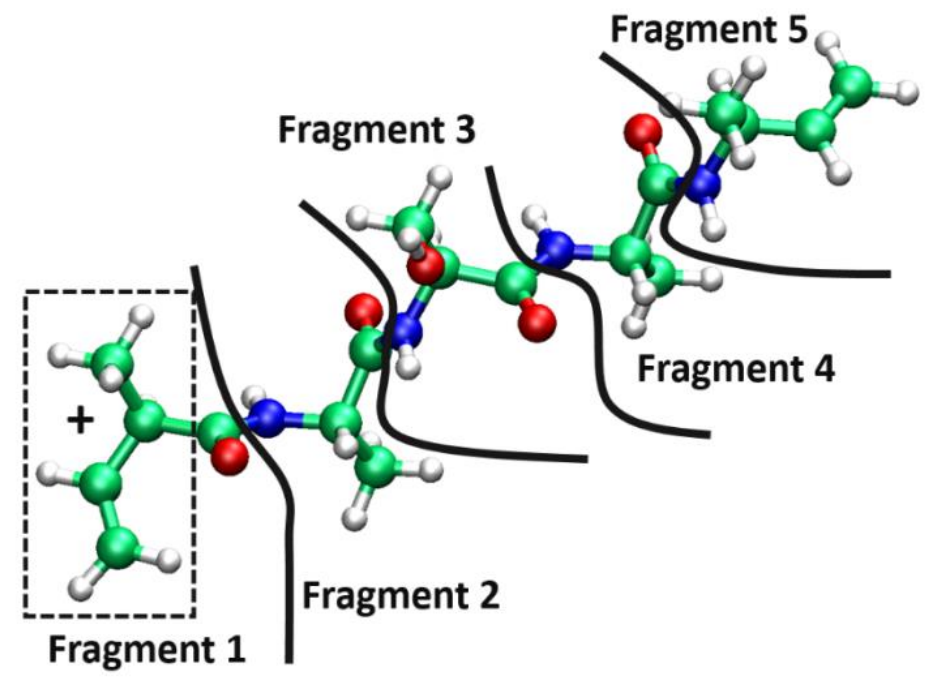

Figure 8: decomposition of the AASAA pentapeptide into fragments.

We start by the discussion of Fragment 1 . All the curves are very similar, especially during the first $5 \mathrm{fs}$. The charge, initially around 0.86 , drops to 0.65 in average with superimposed oscillations. These oscillations indicate charge migrations toward the peptide backbone. After this initial period, we notice slight deviations among the curves. If we consider the light blue curve as the richest auxiliary basis set, reference (BS2), we see that BS1 (GEN-A2 on all atoms) and BS5 (GEN-A3 on all atoms) are the basis sets showing the largest, though moderate, deviations. The other curves are very similar. In addition, we note that even for BS2 and BS5, the variations with respect to BS2 consist in a vertical shift of the curves, leaving the overall structuration of the oscillations unchanged. This is a quite encouraging result indicating that the underlying charge migration phenomena are correctly captured by all auxiliary basis sets. When considering charge fluctuations on Fragments 2 and 3, similar conclusions prevail. 

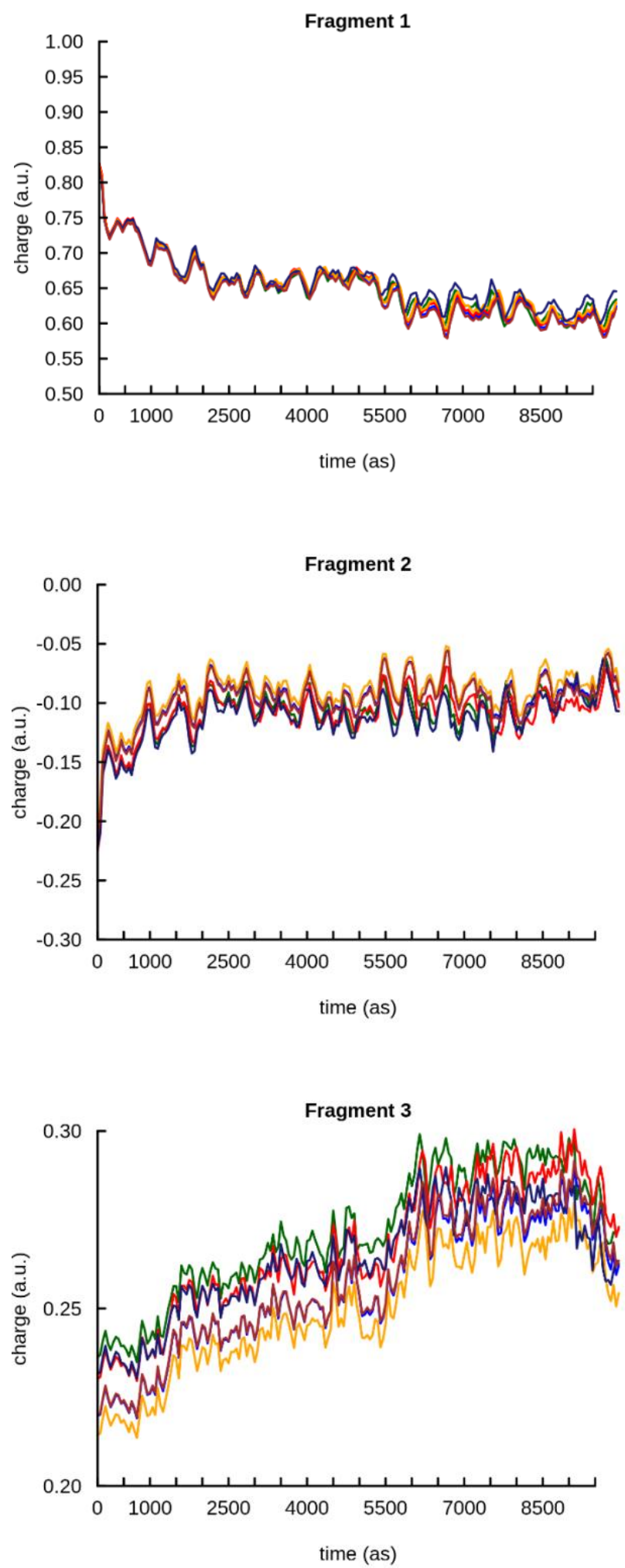

Figure 9: Charge evolution for Fragment 1 to 3 after release of the potential constrain. Color code: BS1 (green), BS2 (blue), BS3 (red), BS4 (orange); BS5 (dark blue), BS6 (brown). Note the different scales for each graph. 


\section{CONCLUSIONS}

In this article we have extensively assessed the reliability of Auxiliary DFT for carrying out electronic dynamic simulations by RT-TD-DFT. We also took the opportunity of this work to test the recently proposed MINRES algorithm to deal with very large systems comprised with more than one hundred thousand auxiliary basis functions.

The methodology has been tested on various typical applications of RT-TD-DFT, namely the calculation of absorption spectra in the gas phase or in solvent by a hybrid QM/MM (Quantum Mechanics/Molecular Mechanics) scheme, the calculation of electronic stopping power curves for proton in lithium cluster, the deposition of energy upon collision of a zeolite by a swift proton, and finally on the investigation of charge migrations within an ionized peptide. We have obtained very encouraging results. Firstly, we highlighted the reliability of the ADFT framework for electronic dynamics simulations. In all our test examples, we could hardly find evident artefacts due to this formalism. Secondly, we showed that even the GEN-A2 auxiliary basis provides very satisfactory results. Overall, we have demonstrated that the RT-TD-ADFT is a very a safe and powerful strategy to tackle electron dynamics simulations in small, large or very large molecular systems and their environment.

\section{ACKNOWLEDGMENTS}

We are grateful to Fabien Bruneval and Ivan Maliyov for providing us with the structure of the Lithium system, to Tzonka Mineva for the zeolite coordinates and to Laura Berstis for the peptide coordinate. This work was supported by the Agence nationale de la Recherche scientifique (Project RUBI, number: ANR-19-CE29-0011-01). This work was performed using HPC resources from the GENCI (CINES/IDRIS, Grant No. 2020- A0080806830).

\section{REFERENCES}

1. Andrade X, Strubbe D, De Giovannini U, Larsen AH, Oliveira MJT, Alberdi-Rodriguez J, Varas A, Theophilou I, Helbig N, Verstraete MJ, Stella L, Nogueira F, Aspuru-Guzik A, Castro A, Marques MAL, Rubio A (2015) Real-space grids and the Octopus code as tools for the development of new simulation approaches for electronic systems. Phys Chem Chem Phys 17:31371-31396 . https://doi.org/10.1039/C5CP00351B

2. Wopperer P, Dinh PM, Reinhard PG, Suraud E (2015) Electrons as probes of dynamics in molecules and clusters: A contribution from Time Dependent Density Functional Theory. Phys Rep 562:1-68 . http://dx.doi.org/10.1016/j.physrep.2014.07.003 
3. Runge E, Gross EKU (1984) Density-Functional Theory for Time-Dependent Systems. Phys Rev Lett 52:997-1000 . https://doi.org/10.1103/PhysRevLett.52.997

4. CASIDA ME (1995) Time-Dependent Density Functional Response Theory for Molecules. In: Recent Advances in Density Functional Methods. WORLD SCIENTIFIC, pp 155-192

5. Yabana K, Bertsch GF (1996) Time-dependent local-density approximation in real time. Phys Rev B 54:4484-4487 . https://doi.org/10.1103/PhysRevB.54.4484

6. Calvayrac F, Reinhard PG, Suraud E (1995) Nonlinear plasmon response in highly excited metallic clusters. Phys Rev B 52:R17056-R17059 . https://doi.org/10.1103/PhysRevB.52.R17056

7. A. Schleife, E. W. Draeger, V. M. Anisimov, A. A. Correa, Y. Kanai (2014) Quantum Dynamics Simulation of Electrons in Materials on High-Performance Computers. Computing in Science \& Engineering 16:54-60 . https://doi.org/10.1109/MCSE.2014.55

8. Tavernelli I, Röhrig UF, Rothlisberger U (2005) Molecular dynamics in electronically excited states using time-dependent density functional theory. Molecular Physics 103:963-981 . https://doi.org/10.1080/00268970512331339378

9. Li X, Smith SM, Markevitch AN, Romanov DA, Levis RJ, Schlegel HB (2005) A time-dependent Hartree-Fock approach for studying the electronic optical response of molecules in intense fields. Phys Chem Chem Phys 7:233-239. https://doi.org/10.1039/b415849k

10. Lopata K, Govind N (2011) Modeling Fast Electron Dynamics with Real-Time Time-Dependent Density Functional Theory: Application to Small Molecules and Chromophores. J Chem Theor Comput 7:1344-1355 . https://doi.org/10.1021/ct200137z

11. Maliyov I, Crocombette J-P, Bruneval F (2018) Electronic stopping power from time-dependent density-functional theory in Gaussian basis. The European Physical Journal B 91:172 . https://doi.org/10.1140/epjb/e2018-90289-y

12. Nguyen TS, Parkhill J (2015) Nonadiabatic Dynamics for Electrons at Second-Order: Real-Time TDDFT and OSCF2. J Chem Theor Comput 11:2918-2924. https://doi.org/10.1021/acs.jctc.5b00262

13. Li X, Govind N, Isborn C, DePrince AE, Lopata K (2020) Real-Time Time-Dependent Electronic Structure Theory. Chem Rev 120:9951-9993 . https://doi.org/10.1021/acs.chemrev.0c00223

14. Valiev M, Bylaska EJ, Govind N, Kowalski K, Straatsma TP, Van Dam HJJ, Wang D, Nieplocha J, Apra E, Windus TL, de Jong WA (2010) NWChem: A comprehensive and scalable open-source solution for large scale molecular simulations. Comput Phys Commun 181:1477-1489 . http://dx.doi.org/10.1016/j.cpc.2010.04.018

15. Dunlap BI, Rösch N, Trickey SB (2010) Variational fitting methods for electronic structure Molecular Physics 108:3167-3180 https://doi.org/10.1080/00268976.2010.518982

16. Köster, A.M, Geudtner, G, Álvarez-Ibarra, A, Calaminici, P, Casida, M.E, Carmona-Espíndola, J, Delesma, F.A., Delgado-Venegas, R., Domínguez, V.D., Flores-Moreno, R, Gamboa, G.U, Goursot, A, Heine, Th., Ipatov, A., de la Lande, Aurélien, Janetzko, F., Martín del Campo, J., PedrozaMontero, J.N., Petterson, L.G.M.;, Mejía-Rodríguez, D, Reveles, J., Vásquez-Pérez, J, Vela, A, Zúñiga-Gutiérrez, B, Salahub, DR (2020) deMon2k. Mexico City 
17. Wu X, Teuler J-M, Cailliez F, Clavaguéra C, Salahub DR, de la Lande A (2017) Simulating Electron Dynamics in Polarizable Environments. J Chem Theory Comput 13:3985-4002 . https://doi.org/10.1021/acs.jctc.7b00251

18. Köster AM, Reveles JU, del Campo JM (2004) Calculation of exchange-correlation potentials with auxiliary function densities. J Chem Phys 121:3417-3424 . https://doi.org/10.1063/1.1771638

19. Mejía-Rodríguez D, Trickey SB (2021) Variational properties of auxiliary density functionals. Theoretical Chemistry Accounts 140:37 . https://doi.org/10.1007/s00214-021-02731-2

20. Calaminici $P$, Alvarez-Ibarra A, Cruz-Olvera D, Domínguez-Soria V-D, Flores-Moreno R, Gamboa GU, Geudtner G, Goursot A, Mejía-Rodríguez D, Salahub DR, Zuniga-Gutierrez B, Köster A (2016) Auxiliary Density Functional Theory: From Molecules to Nanostructures. In: Leszczynski J (ed) Handbook of Computational Chemistry. Springer Netherlands, Dordrecht, pp 1-67

21. A. Alvarez-Ibarra, A. M. Köster (2013) Double asymptotic expansion of three-center electronic repulsion integrals. J Chem Phys 139:024102 . https://doi.org/10.1063/1.4812183

22. Pedroza-Montero JN, Morales JL, Geudtner G, Álvarez-Ibarra A, Calaminici P, Köster AM (2020) Variational Density Fitting with a Krylov Subspace Method. J Chem Theory Comput 16:2965-2974 . https://doi.org/10.1021/acs.jctc.9b01212

23. Víctor D. Domínguez-Soria, Gerald Geudtner, José Luis Morales, Patrizia Calaminici, Andreas M. Köster (2009) Robust and efficient density fitting. J Chem Phys 131:124102 . https://doi.org/10.1063/1.3216476

24. Alvarez-Ibarra A, Parise A, Hasnaoui K, de la Lande A (2020) The physical stage of radiolysis of solvated DNA by high-energy-transfer particles: insights from new first principles simulations. Phys Chem Chem Phys 22:7747-7758 . https://doi.org/10.1039/D0CP00165A

25. Li X, Tully JC, Schlegel HB, Frisch MJ (2005) Ab initio Ehrenfest dynamics. J Chem Phys 123:084106 . https://doi.org/10.1063/1.2008258

26. Magnus W (1954) On the exponential solution of differential equations for a linear operator. Commun Pure App Math 7:649-673 . https://doi.org/10.1002/cpa.3160070404

27. Köster AM, Flores-Moreno R, Reveles JU (2004) Efficient and reliable numerical integration of exchange-correlation energies and potentials. J Chem Phys 121:681-690. https://doi.org/doi:http://dx.doi.org/10.1063/1.1759323

28. Mintmire JW, Dunlap BI (1982) Fitting the Coulomb potential variationally in linear-combinationof-atomic-orbitals density-functional calculations. Phys Rev A 25:88-95

29. BROYDEN CG (1970) The Convergence of a Class of Double-rank Minimization Algorithms 1. General Considerations. IMA Journal of Applied Mathematics 6:76-90 . https://doi.org/10.1093/imamat/6.1.76

30. BROYDEN CG (1970) The Convergence of a Class of Double-rank Minimization Algorithms: 2. The New Algorithm. IMA Journal of Applied Mathematics 6:222-231. https://doi.org/10.1093/imamat/6.3.222

31. Fletcher R (1970) A new approach to variable metric algorithms. The Computer Journal 13:317322 . https://doi.org/10.1093/comjnl/13.3.317 
32. Pedroza-Montero JN, Delesma FA, Morales JL, Calaminici P, Köster AM (2020) Variational fitting of the Fock exchange potential with modified Cholesky decomposition. J Chem Phys 153:134112 . https://doi.org/10.1063/5.0020084

33. Mejía-Rodríguez D, Köster AM (2014) Robust and efficient variational fitting of Fock exchange. J Chem Phys 141:124114 . https://doi.org/doi:http://dx.doi.org/10.1063/1.4896199

34. Delesma FA, Geudtner G, Mejía-Rodríguez D, Calaminici P, Köster AM (2018) Range-Separated Hybrid Functionals with Variational Fitted Exact Exchange. J Chem Theor Comput 14:5608-5616 . https://doi.org/10.1021/acs.jctc.8b00436

35. R Development Core Team (2012) R: A Language and Environment for Statistical Computing. R Foundation for Statistical Computing, Vienna, Austria

36. Köster AM (2003) Hermite Gaussian auxiliary functions for the variational fitting of the Coulomb potential in density functional methods. J Chem Phys 118:9943-9951. https://doi.org/doi:http://dx.doi.org/10.1063/1.1571519

37. Perdew JP, Burke K, Ernzerhof M (1996) Generalized Gradient Approximation Made Simple. Phys Rev Lett 77:3865-3868

38. Blackford LS, Choi J, Cleary AJ, D'Azevedo EF, Demmel J, Dhillon IS, Dongarra J, Hammarling S, Henry G, Petitet A, Stanley K, Walker DW, Whaley RC (1997) ScaLAPACK: A Linear Algebra Library for Message-Passing Computers

39. Andreas M. Köster, Jorge M. del Campo, Florian Janetzko, Bernardo Zuniga-Gutierrez (2009) A MinMax self-consistent-field approach for auxiliary density functional theory. J Chem Phys 130:114106 . https://doi.org/10.1063/1.3080618

40. Calaminici P, Janetzko F, Köster AM, Mejia-Olvera R, Zuniga-Gutierrez B (2007) Density functional theory optimized basis sets for gradient corrected functionals: $3 d$ transition metal systems. J Chem Phys 126:044108 . https://doi.org/doi:http://dx.doi.org/10.1063/1.2431643

41. Calaminici P, Flores-Moreno R, Köster AM (2005) A Density Functional Study of Structures and Vibrations of Ta3O and Ta30-. Computing Letters 1:164-171. https://doi.org/10.1163/157404005776611420

42. de la Lande, Aurélien, Köster, Andreas M, Denisov, Sergey, Mostafavi, Mehran (2021) The Mystery of Sub-Picosecond Charge Transfer Following Irradiation of Hydrated Uridine Monophosphate. under review:

43. Maliyov I, Crocombette J-P, Bruneval F (2020) Quantitative electronic stopping power from localized basis set. Phys Rev B 101:035136 . https://doi.org/10.1103/PhysRevB.101.035136

44. Laura Berstis, Gregg T. Beckham, Michael F. Crowley (2015) Electronic coupling through natural amino acids. J Chem Phys 143:225102 . https://doi.org/10.1063/1.4936588

45. Řezáč J, Lévy B, Demachy I, de la Lande A (2012) Robust and Efficient Constrained DFT Molecular Dynamics Approach for Biochemical Modeling. J Chem Theor Comput 8:418-427 . https://doi.org/10.1021/ct200570u

46. Cheng C-L, Evans JS, Van Voorhis T (2006) Simulating molecular conductance using real-time density functional theory. Phys Rev B 74:155112 
